\title{
Performance Evaluation of Routing Protocol (RPL) for Internet of Things
}

\author{
Qusai Q. Abuein, Muneer Bani Yassein, Mohammed Q. Shatnawi, Laith Bani-Yaseen, Omar Al-Omari, Moutaz \\ Mehdawi and Hussien Altawssi \\ Faculty of Computer and Information Technology \\ Jordan University of Science and Technology \\ Irbid, Jordan
}

\begin{abstract}
Recently, Internet Engineering Task Force (IETF) standardized a powerful and flexible routing protocol for Low Power and Lossy Networks (RPL). RPL is a routing protocol for low power and lossy networks in the Internet of Things. It is an extensible distance vector protocol, which has been proposed for low power and lossy networks in the global realm of IPv6 networks, so it selects the routes from a source to a destination node based on certain metrics injected into the objective function (OF). There has been an investigation of the performance of RPL in the lighter density network. This study investigates the performance of RPL in medium density using of two objective function in various topologies (e.g. grid, random). The performance of RPL is studied using various metrics. For example, Packet Delivery Ratio (PDR), Power Consumption and Packet Reception Ratio (RX) using a fixed Packet Reception Ratio (RX) values.
\end{abstract}

Keywords-density network; objective function; zero grid; packet delivery; power consumption; Internet of Things

\section{INTRODUCTION}

Internet of Things (IoT) is a technology in which everyday objects form an Internet network through where they can communicate with each other. The Internet of Things is a huge network of things or objects that can be embedded with a unique ID which then allows it to be connected to the internet, this huge network allows the devices to exchange data simultaneously for its specific purpose. The IoT allows the object to sense and collects data in the existing network infrastructure, which then will create opportunities for the realtime integration between the Machines and the physical world, this will result in economic benefit, improved accuracy and efficiency. The Internet Engineering Task Force (IETF) STANDARDIZED a powerful and flexible Routing Protocol for Low Power and Lossy Networks (RPL). It selects the ideal routes from a source to a destination node based on certain metrics injected into the Objective Function (OF). Previous studies. Many previous studies have investigated the performance of the $\mathrm{OFO}$ and MRHOF objective functions in the light density network. This study will investigate the performance of the two OFs using various metrics like Packet Delivery Ratio, Energy Consumption in the medium density network. In this study, the performance of RPL will be investigated in terms of two Objective Functions under two topologies (grid, random) which make this work distinctive. To study the RPL performance, various metrics are considered Packet Delivery Ratio (PDR), Power Consumption and RX.
The evaluation will be conducted based on these parameters (RX, topology) and compared for both OFs within a medium density network. In [1], Objective function Zero is the default Objective function in the Routing Protocol for Low-Power and Lossy Networks, OF0 is simple, it selects its parent depending on the minimum ranks of the neighbors. The node rank is usually an integer, it represents the nodes location. The most common objective function in RPL is Of0, This objective function permits the upward traffic to be routed through the selected parent (preferred parent) without performing any load balancing. In [2], Minimum Rank with hysteresis Objective Function (MRHOF) was proposed, it is commonly used for metrics as it is based on metric-containers, the container is used to determine the features and the nature of routing objects, this objective function the path cost is equal to the cost of the selected metric, from a child node to the sink node through its neighbors. The route cost is calculated by the node by adding the two components, the cost of the nominated measurement and the selected measurement for the connection to a nominee neighbor.

\section{RELATED WORK}

The growing attention of the research and industrial communities towards RPL is sworn from the amount of the recently published research, where RPL performance has been studied under the umbrella of different contexts and platforms. The authors of [3-5] show the effectiveness of RPL pertaining to exiguous delay, quick configuration, and self-healing. RPL is a Distance Vector IPv6 routing protocol designed for Low Sensor Networks, it is specifically designed represents the building of Destination Oriented Directed Acyclic Graph (DODAG) using OF0 or MRHOF with a set of constraints/metrics, the purpose is to calculate the best path, the node can operate with multiple OFS concurrently because the distribution varies greatly in different network topologies and different objectives may need to transmit traffic with different necessities of route superiority. The objective function does not require the metric and restrictions however does impose some rules to form the DODAG. One of the responsibilities of the network layer is delivering packets to the destination nodes via multiple hops separating the source node from the destination node. The routing table allows the packet to gain knowledge of the next hop neighbor node, the routing tables is populated by routing protocols. RPL builds a logical routing topology graph which is constructed ended a physical network to come across a assured measures and the network supervisor decides to have 
multiple direction-finding topologies operating at the same time used to transmit the stream of traffic with multiple set of requests. Any node in the network can join one or more graphs, in this case they are called RPL occurrences and label the traffic tolerating to the graph characteristics.

The authors [9] provide the comparison for both OFs performances in a light density network under two different topologies (grid, random). RPL supports peer-to-peer communication which means any node in the graph in communicate with any other node in the same graph. When a node communicates with another node in the LLN network, the packet moves 'upwards' to a parent and 'downwards' to the destination.

\section{PERFORMANCE EVALUATION}

The main point of this study is to investigate the performance of the RPL in terms of OFs under two different topologies. A comparative study of broadcast mechanisms of RPL in IoT in conducted. Broadcast mechanisms using the rooted DAG-like logical structure maintained by the unicast routing protocol in RPL will be introduced and their performance will be studied in order to create a new broadcast mechanism with self-pruning to make the RPL OFs performance more efficient.

\section{A. Results and Deduction}

The experiments are conducted under the medium density network which consists of (50, 65, 75 and 85) nodes using random and grid topologies and with a Fixed RX=60.The RPL behavior in terms of power consumption and packet delivery ratio and is investigated. The OF0 was installed and results were obtained.

Figure 2 shows the behavior of the PDR based on a fixed value of $\mathrm{RX}=60$ and a various number of nodes for the grid and the random topology using the objective function OF0. In the Grid topology, the PDR increased between the 50-65 nodes but it decreased between the 75-85 nodes, this shows that the PDR is more efficient when using the OF0 in the grid topology when the density is between 50-65 nodes. In the Random topology, the PDR increased between the 50-65 nodes, the PDR also increased at 75 nodes and above, this shows that the PDR is more efficient when using the OF0 in the random topology when the density is between 50-65 nodes and above 75 nodes.

\section{PDR USING OF0 IN GRID}

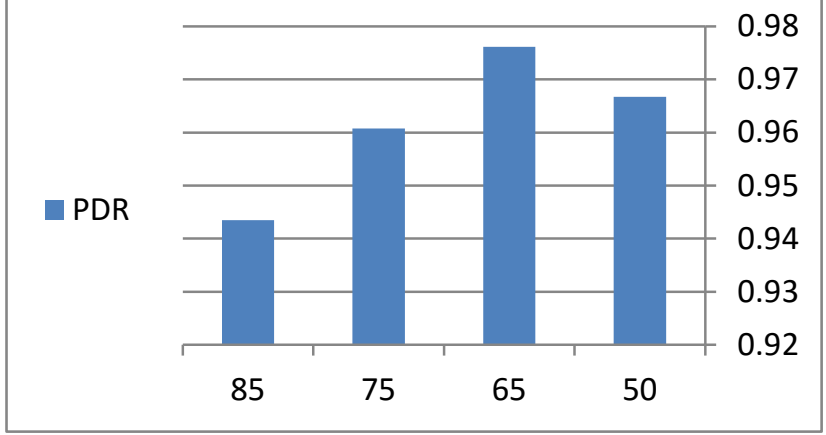

Fig. 1. Values of PDR in GRID Topology using OFO

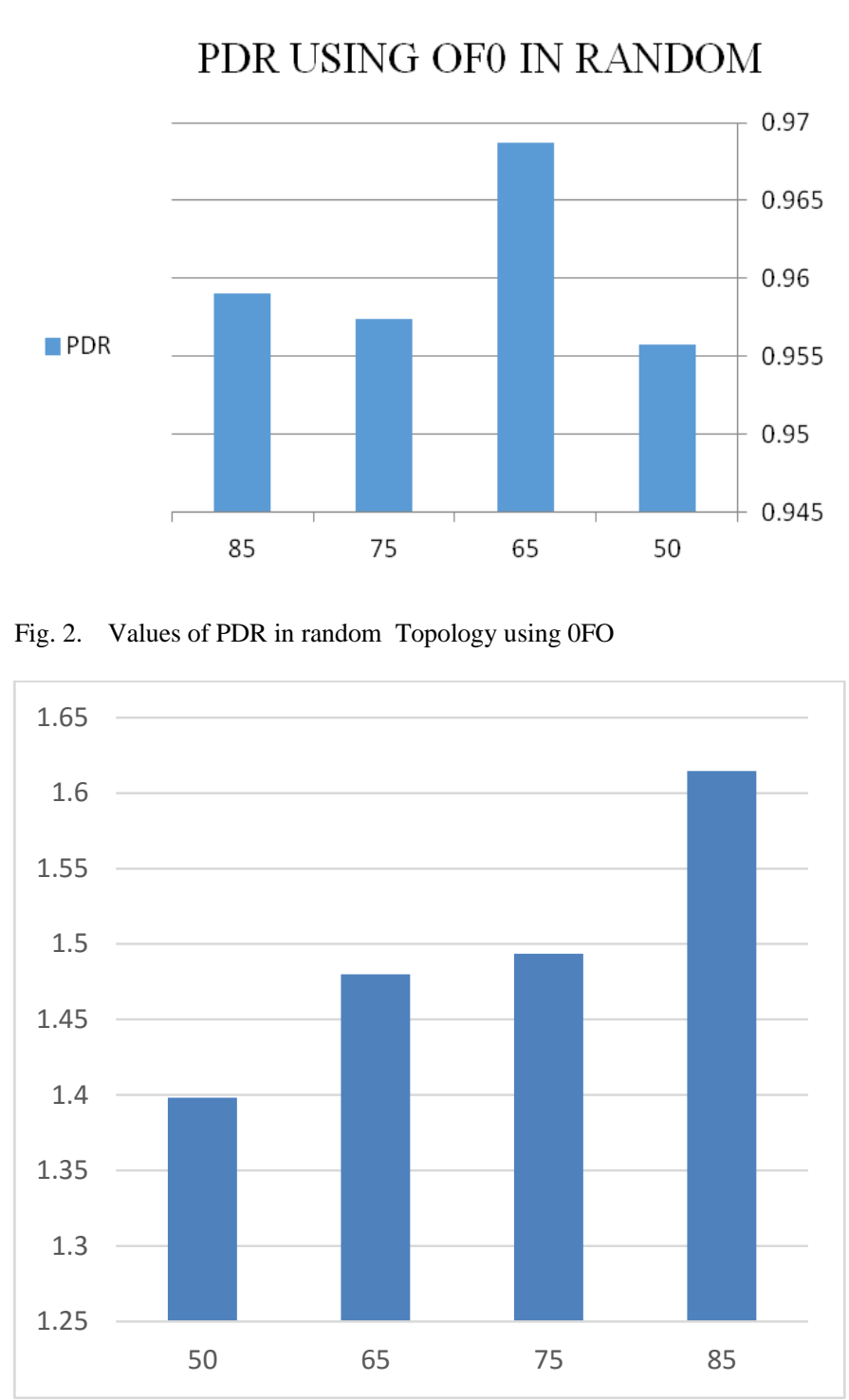

Fig. 3. Power Consumption Using OF0 in Grid

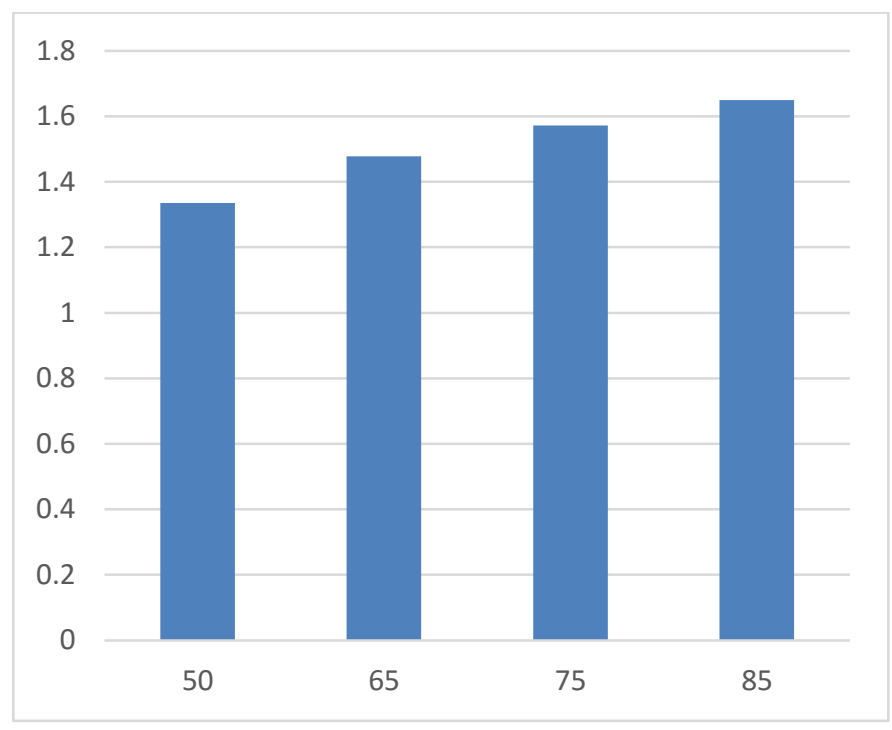

Fig. 4. Power Consumption Using Of0 in Random Topology 


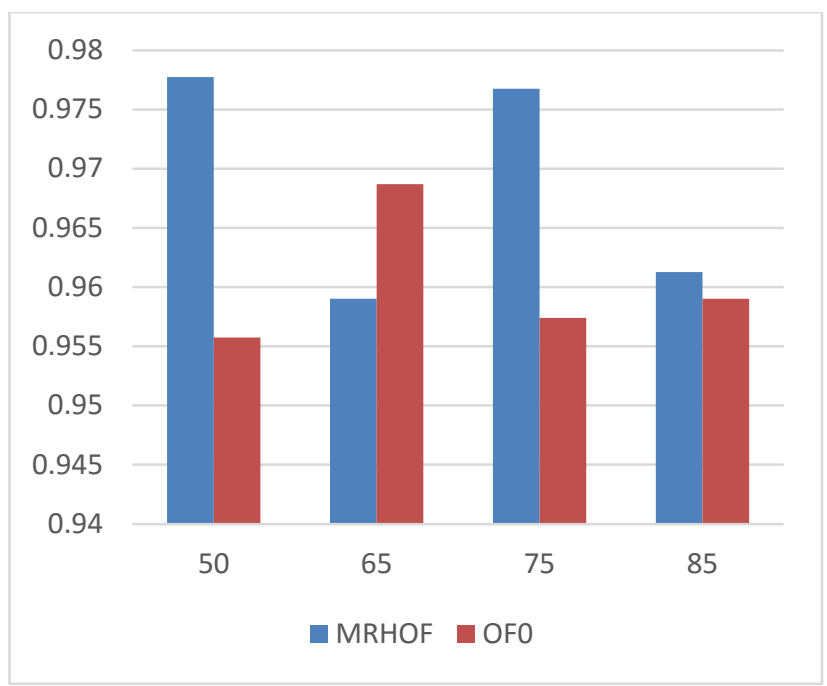

Fig. 5. Values of PDR with OF0 and MRHOF in random topology

Figure 5 shows the presentation of the power depletion based on a fixed value of $\mathrm{RX}=60$ and a various number of nodes for the grid and the random topology using the objective function MRHOF. In the Grid topology, the Power Consumption increased gradually as the number of nodes increased, this shows that the Power Consumption is not efficient when using the MRHOF in the grid topology when the density is between 50-85 nodes. In the Random topology, the Power Consumption increased gradually as the number of nodes increased, the power consumption was almost stable between 65-85 nodes. This shows that the Power Consumption is more efficient to use MRHOF in the random topology when the density is above 50 nodes.

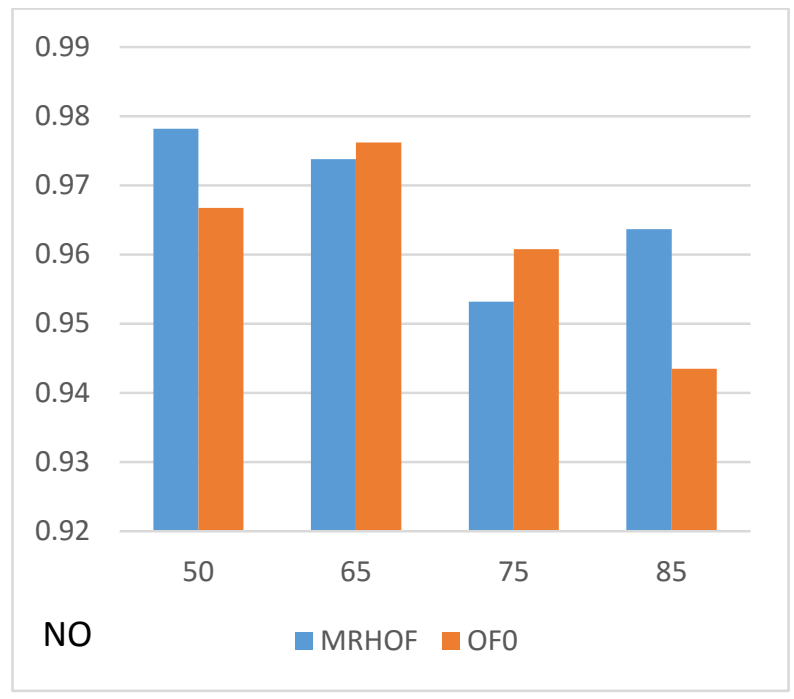

Fig. 6. Values of PDR with OF0 and MRHOF in grid topology
Figure 6 shows that the PDR of Objective Function Zero is roughly $0.956 \%$, and that the PDR of MRHOF is around $0.97 \%$.

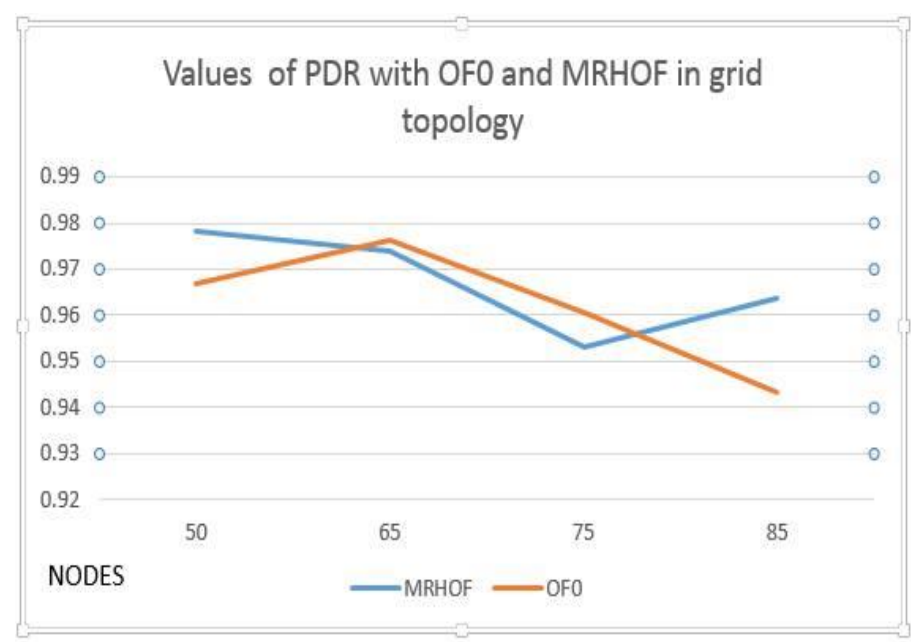

Fig. 7. Values of PDR with Of0 and MRHOF in Grid Topology

In Figure 7, the values of the PDR of MRHOF is approximately $0.97 \%$. The average Packet Delivery Ratio of OF0 decreases as the number of nodes increases.

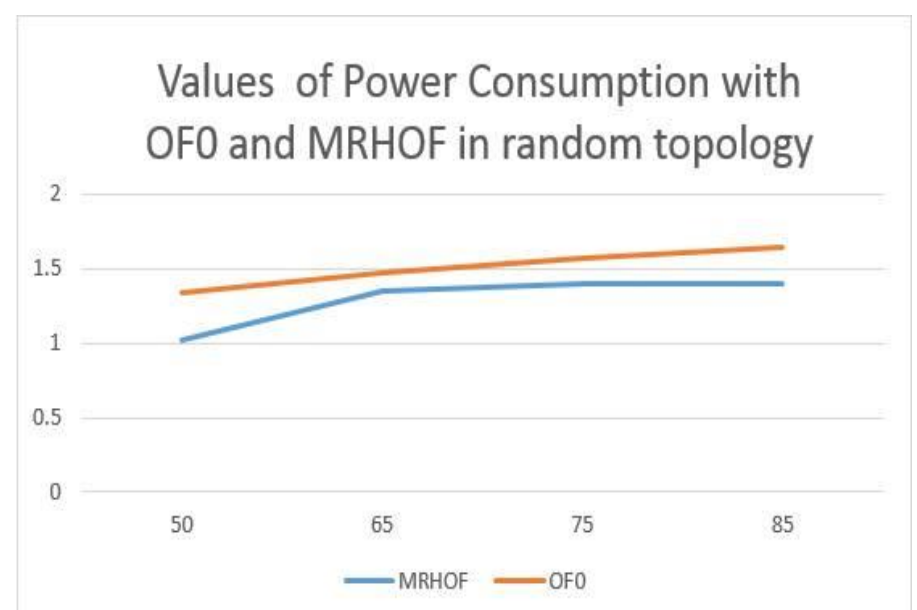

Fig. 8. Values of Power Consumption with OF0 and MRHOF in the Random Topology

In Figure 8, shows the appearance of power depletion with OF0 and MRHOF in the Random Topology, power depletion of MRHOF which is around $1.29 \%$ and the power depletion of OF0 is approximately $1.50 \%$ using Random Topology. Figure 9 shows the behavior of the Power Consumption has almost stable figures for both MRHOF and OF0 when the Packet Reception Ratio $=60 \%$ and in a Medium Density Network. 


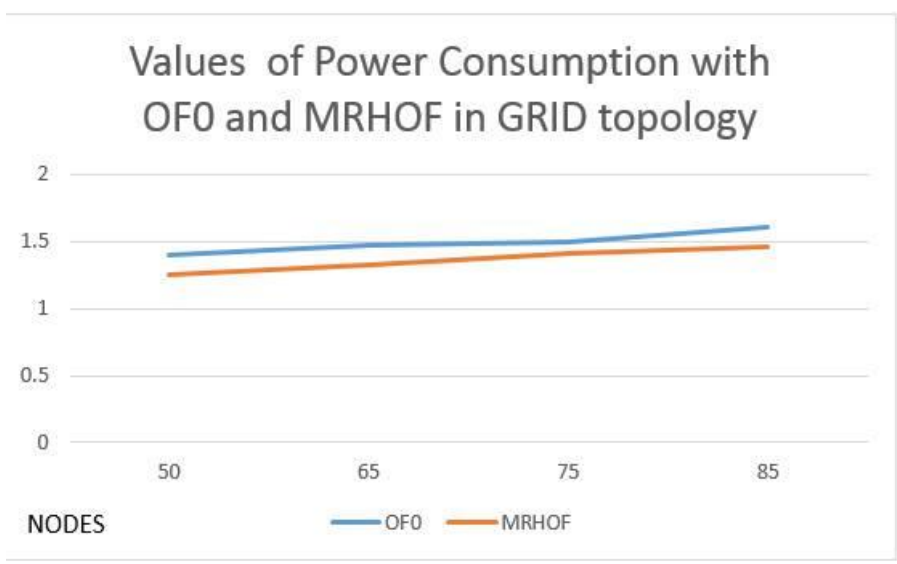

Fig. 9. Values of Power Consumption with OFO and MRHOF in GRID topology

\section{CONCLUSION}

This research proved that the Routing Protocol for Low power and Lossy networks is extremely demanding upon using the Objective function Zero and Minimum Rank hysteresis Objective Functions in terms of Packet Delivery Ratio and Power Consumption in the Medium Density Network. It has been revealed that the Packet Reception Ratio is best when it is equal to $60 \%$ for both Objective functions in the relation to Packet delivery ratio and Power Consumption. The best performance of The Routing Protocol for Low power and Lossy networks performances is at its best when the network density is between $50-65$ motes for the $\mathrm{RX}=60 \%$ in the Grid and Random Topologies.

\section{REFERENCES}

[1] Thubert, "Objective Function Zero," RFC 6552, March 2012.

[2] O. Gnawali and P. Levis, "The ETX Objective Function for RPL," IETF Internet Draft: draft-gnawali-roll-etxof-00, 2010.

[3] N. Accettura, L. Grieco, G. Boggia, and P. Camarda, "Performance Analysis of the RPL Routing Protocol," in Proc. of 2011 IEEE International Conference on Mechatronics, April 2011.
[4] E. Ancillotti, R. Bruno, and M. Conti, "The role of the RPL routing protocol for smart grid communications," IEEE Communications Magazine, vol. 51, no. 1, pp. 75-83.

[5] J. Tripathi, J. de Oliveira, and J. Vasseur, "Applicability Study of RPL with Local Repair in Smart Grid Substation Networks," in 2010 First IEEE International Conference on Smart Grid Communications (SmartGridComm), pp. 262-267. 2010

[6] O. Gnawali and P. Levis, "The Minimum Rank with Hysteresis Objective Function", RFC 6719 (Proposed Standard), Internet Engineering Task Force, Sep, 2012.

[7] O. Gaddour and A. Koubaa. RPL in a nutshell: A survey. Comput. Netw. pp. 3163-3178, 2012.

[8] Gaddour, O., Koubaa, A. Chaudhry, S. Tezeghdanti, M., Chaari, R., Abid, M.: Simulation and performance evaluation of DAG construction with RPL. In: 2012 Third International Conference on Communications and Networking (ComNet), pp. 1-8, 2012.

[9] Mamoun Qasem, Hussien Altawssi, Muneer BaniYassien, Ahmed AlDubai. Performance Evaluation of RPL Objective Functions,2015.

[10] Reactive Discovery of Point-to-Point Routes in Low Power and Lossy Networks - http://tools.ietf.org/html/draft-ietf-roll-p2p-rpl.

[11] Gaddour, O., Koubaa, A., Chaudhry, S., Tezeghdanti, M., Chaari, R., Abid, M.: Simulation and performance evaluation of DAG construction with RPL. In: 2012 Third International Conference on Communications and Networking (ComNet),pp. 1-8 (2012).

[12] M. Vucinic, B. Tourancheau, and A. Duda "Performance comparison of the rpl and loadng routing protocols in a home automation scenario." Proceedings of IEEE WCNC, 2013.

[13] L.Songhua, W. Muqing, C. Chuanfeng, L. Bo and L. Simu. "A highthroughput routing metric for multi-hop Ad hoc networks based on real time testbed." TENCON 2013,Oct. Mar,2013,pp.1-4.

[14] P. Gonizzi, R. Monica, and G. Ferrari. "Design and evaluation of a delay-efficient RPL routing metric." Wireless Communications and Mobile Computing Conference (IWCMC), 2013 9th International. IEEE,2013.

[15] N. Tsiftes, J. Eriksson, N. Finne, O. Fredrik, J. Höglund, A. Dunkels, A Framework for Low-Power IPv6 Routing Simulation, Experimentation, and Evaluation, SIGCOMM'10, New Delhi, India, November 2010, pp. 479-480.

[16] M. Nuvolone, "Stability analysis of the delays of the routing protocol over low power and lossy networks," Master's thesis, KTH RIT, 2010.

[17] W. Xie, M. Goyal, H. Hosseini, J. Martocci, Y. Bashir, E. Baccelli, A. Durresi, "A performance analysis of point-to-point routing along a directed acyclic graph in low power and lossy networks", 2010. 